\title{
BMJ Open Acupuncture point injection treatment of primary dysmenorrhoea: a randomised, double blind, controlled study
}

\author{
C Wade,${ }^{1,2}$ L Wang, ${ }^{3}$ W J Zhao, ${ }^{3}$ F Cardini, ${ }^{4}$ F Kronenberg, ${ }^{1,5}$ S Q Gui, ${ }^{3}$ Z Ying, ${ }^{6}$ \\ N Q Zhao, ${ }^{7}$ M T Chao, ${ }^{1,8} \mathrm{~J} \mathrm{Yu}^{3}$
}

To cite: Wade $C$, Wang $L$, Zhao WJ, et al. Acupuncture point injection treatment of primary dysmenorrhoea: a randomised, double blind, controlled study. BMJ Open 2016:6:e008166

doi:10.1136/bmjopen-2015008166

- Prepublication history for this paper is available online. To view these files please visit the journal online (http://dx.doi.org/10.1136/ bmjopen-2015-008166).

Received 13 March 2015 Revised 25 November 2015 Accepted 26 November 2015

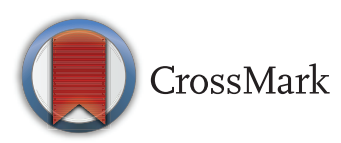

For numbered affiliations see end of article.

Correspondence to

C Wade;

wade@columbia.edu

\section{ABSTRACT}

Objective: To determine if injection of vitamin K3 in an acupuncture point is optimal for the treatment of primary dysmenorrhoea, when compared with 2 other injection treatments.

Setting: A Menstrual Disorder Centre at a public hospital in Shanghai, China.

Participants: Chinese women aged 14-25 years with severe primary dysmenorrhoea for at least 6 months not relieved by any other treatment were recruited. Exclusion criteria were the use of oral contraceptives, intrauterine devices or anticoagulant drugs, pregnancy, history of abdominal surgery, participation in other therapies for pain and diagnosis of secondary dysmenorrhoea. Eighty patients with primary dysmenorrhoea, as defined on a 4-grade scale, completed the study. Two patients withdrew after randomisation.

Interventions: A double-blind, double-dummy, randomised controlled trial compared vitamin $\mathrm{K} 3$ acupuncture point injection to saline acupuncture point injection and vitamin K3 deep muscle injection.

Patients in each group received 3 injections at a single treatment visit.

Primary and secondary outcome measures: The primary outcome was the difference in subjective perception of pain as measured by an 11 unit Numeric Rating Scale (NRS). Secondary measurements were Cox Pain Intensity and Duration scales and the consumption of analgesic tablets before and after treatment and during 6 following cycles.

Results: Patients in all 3 groups experienced pain relief from the injection treatments. Differences in NRS measured mean pain scores between the 2 active control groups were less than 1 unit $(-0.71, \mathrm{Cl}-1.37$ to -0.05 ) and not significant, but the differences in average scores between the treatment hypothesised to be optimal and both active control groups $(1.11, \mathrm{Cl}$ 0.45 to 1.78$)$ and (1.82, Cl 1.45 to 2.49 ) were statistically significant in adjusted mixed-effects models. Menstrual distress and use of analgesics were diminished for 6 months post-treatment.

Conclusions: Acupuncture point injection of vitamin $\mathrm{K} 3$ relieves menstrual pain rapidly and is a useful treatment in an urban outpatient clinic.

\section{Strengths and limitations of this study}

- The results of this trial document the efficacy of a treatment in use for over 40 years at a highvolume Menstrual Disorder Centre and provide important information about a treatment for a common condition of women worldwide.

- The design is rigorous: a double-blind, doubledummy, randomised controlled trial powered to compare three active treatments.

- The primary outcome effect size of the difference between groups is robust.

- Owing to funding limitations, the study was implemented in two phases under two different randomisation schemes and data from the first phase were analysed prior to the implementation of the second phase.

- Stratified analyses were conducted to account for any differences between the results of the two phases of the trial.

Trial registration number: NCT00104546; Results.

\section{INTRODUCTION}

Dysmenorrhoea is highly prevalent and a major cause of activity restriction and school absence among young women worldwide. ${ }^{12}$ Primary dysmenorrhoea is a painful menstrual cramping of the uterus without identifiable organic pathology that affects as many as $85 \%$ of women, $20 \%$ of whom describe their pain as severe. ${ }^{3} 4$ The initial onset of primary dysmenorrhoea is usually shortly after menarche, 6-12 months after ovulatory cycles begin. High levels of uterine prostaglandins, a hormone associated with uterine cramping, abdominal pain and contraction of muscular tissue, have been identified as a substantial contributing factor to the pain 
and distress that are the major symptoms of dysmenorrhoea. ${ }^{5}{ }^{6}$ The pain duration is commonly $8-72 \mathrm{~h}$ and is usually associated with the onset of menstrual flow. ${ }^{1}$ The condition often results in activity restriction over 1-3 days of each menstrual cycle, ${ }^{3}$ and therefore the annual costs in terms of disability, in addition to treatment, are high.

The two most common treatments in the USA for dysmenorrhoea are non-steroidal anti-inflammatory drugs (NSAIDs) and oral contraceptives (OCs). ${ }^{7}$ NSAIDs are effective for some but not all women, with a failure rate of up to $25 \% .{ }^{8}$ Both treatments require multiple or daily dosing and have side effects. In China, common treatments for dysmenorrhoea include acupuncture and herbal preparations, with increased use of NSAIDs in recent years; OCs are seldom prescribed for the condition.

Injection of an agent, usually a herbal extract or drug, into an acupuncture point is an innovation in Traditional Chinese Medicine (TCM $)^{69}$ and has been used in China over the past 40 years. ${ }^{10}$ Classical and modern acupuncture usually involves needles inserted at multiple points and left in place for 20-60 min. In contrast, acupuncture point injection is easily administered and standardised, and requires less time at the clinical encounter. ${ }^{11}$

Acupuncture point injection of vitamin K3 has been used as a standard of care treatment for dysmenorrhoea at the Menstrual Disorder Centre at the Obstetrics and Gynecology Hospital in Shanghai, People's Republic of China, since at least $1985,{ }^{12}$ and several uncontrolled studies of the treatment have been conducted in China and Italy with various forms of vitamin $\mathrm{K}^{13-15}$ While vitamin $\mathrm{K} 3$ has been administered consistently at the clinic where this study was conducted, ${ }^{16}$ this form of vitamin $\mathrm{K}$ is not typically used in the USA and Europe. Vitamin K1, however, is routinely administered to newborns shortly after birth to augment coagulation and is considered to be safe. A pilot study of the acupuncture point injection treatment with a cross-over design substituted vitamin K1 for vitamin K3 and explored the acceptability of the treatment in the USA and the absorption of vitamin K1. ${ }^{17} 18$

While the positive results of these prior studies are promising, it was unknown whether vitamin $\mathrm{K}$ as an agent and the acupuncture point as the site of injection were essential to the treatment's effect and whether together they were an optimal treatment for pain relief of primary dysmenorrhoea as compared with other injection treatments. To address this question, the primary objective of this study was to compare: (1) the effects of vitamin $\mathrm{K}$ injection in a non-acupuncture point, (2) the effects of injection of a non-active substance in the acupuncture point, and (3) the combined effects of vitamin $\mathrm{K}$ injected in the acupuncture point. In addition, we sought to evaluate whether the treatments, given only once, relieve menstrual pain at a variety of time points; and to evaluate the feasibility of conducting an international randomised trial with a comparative effectiveness design. Our secondary outcomes were whether the treatments administered in a single cycle had a carryover effect and decreased menstrual pain, distress and the need for medications over six cycles.

\section{MATERIALS AND METHODS}

Written informed consent was obtained from young women, and assent and parental consent from girls and their families. The study was conducted in accordance with the requirements of Good Clinical Practice.

We conducted a double-blind, double-dummy, randomised controlled trial with three parallel groups:

Group A saline acupuncture point injection: Study participants randomised to this group received normal saline injection at the San Yin Jiao or Spleen 6 (SP6) acupuncture point (both legs) and saline in the right buttock. This served as an active control for vitamin $\mathrm{K}$. Group B vitamin $K$ deep muscle injection: Study participants randomised to this group received a vitamin $\mathrm{K} 3$ injection in the right buttock and saline at a nonacupuncture point near but not SP6 (both legs). The clinicians located the sham point at $1 \mathrm{~cm}$ above Sp6, $1 \mathrm{~cm}$ towards the back of the leg from Sp6, and penetrated the needle $1 \mathrm{~cm}$ beneath the skin. This served as an active control for the acupuncture point injection site and ensured that the patients in this group had the same dose of vitamin $\mathrm{K} 3$ as group $\mathrm{C}$, albeit at a different injection site.

Group C vitamin K acupuncture point injection: Study participants randomised to this group received a vitamin K3 injection at the SP6 point (both legs) and saline in the right buttock. A third injection of saline in the buttock was administered to maintain uniformity of treatment with groups $\mathrm{A}$ and $\mathrm{B}$.

The treatments were administered only once.

The alternative hypothesis to all treatments having similar effects on pain of primary dysmenorrhoea is that pain relief in group $\mathrm{C}$-the treatment most closely aligned with TCM theory and clinical innovations at the Menstrual Disorder Centre-was optimal compared with the other two treatments.

Young Chinese women aged 14-25 years with severe primary dysmenorrhoea for at least 6 months that had not been relieved, or only partially relieved, by any other treatment were recruited for this study. A four-grade scale which grades dysmenorrhoea as absent, mild, moderate or severe based on the presence of cramps, whether or not the cramps were very painful or caused patients to sometimes or always cut back on activities, ${ }^{19}$ was used to screen prospective participants to determine eligibility. Young women enrolled in the study were nulliparous, Mandarin or Shanghainese speaking, with regular menstrual cycles for the past 6 months, and who had a working phone.

Exclusion criteria were diagnosis of secondary dysmenorrhoea, the use of OCs, intrauterine devices or 
anticoagulant drugs, pregnancy, history of abdominal surgery, participation in other therapies for acute or chronic pain and diagnosis of dysmenorrhoea due to any recognised cause, such as pelvic inflammatory disease. In addition, women who had had previous injection treatment with vitamin K in SP6 for any reason or who were intolerant to NSAIDs or who planned to be out of the area during the course of the 7-month study were excluded.

Study participants were recruited from patients who presented with pelvic pain at the Menstrual Disorder Centre at the Obstetrics and Gynecology Hospital, Fudan University, in Shanghai, China. Information sessions were conducted at Fudan University to let young women and providers know about the study at the clinic. Study physicians and staff screened prospective women and girls for eligibility and interest, described the study details and obtained consent. For women under the age of 18, parental consent was also obtained. Diagnosis of primary dysmenorrhoea was confirmed by the study physicians on the basis of history and physical examination. Prospective participants who were not currently in pain signed consent forms and were told to return at the onset of their next painful menstrual cycle. Participants could also consent and receive the treatment on their initial visit if they were currently in pain due to menses. Participants were informed that they could withdraw from the study at any time.

Treatments were administered only once by medical doctors who were also experienced acupuncturists. Pain measurement was conducted by clinic staff and not the study physicians. Participants were followed for 6 months with follow-up phone interviews by clinic staff. Treatment consisted of an injection at each of three sites as per group assignment. A single injection of vitamin $\mathrm{K} 3$ is $4 \mathrm{mg} / \mathrm{mL}$, so since participants were injected once in each leg, the total dose was $8 \mathrm{mg}$ of vitamin $\mathrm{K} 3$ or $2 \mathrm{~mL}$ of saline solution. The SP6 acupuncture point is located on the lower leg, 3 'cun' or four widths of the middle phalange of the index finger on the patient, proximal to the peak of the medial malleolus, just along the posterior aspect of the tibial bone. The muscle injected was $m$. soleus (see figure 1 ). An injection of $8 \mathrm{mg}$ of vitamin $\mathrm{K} 3$ or $2 \mathrm{~mL}$ saline (depending on the group) was administered as a deep intramuscular injection in the glutaeal muscle of the right buttock. NSAIDs were available in case any patient was not satisfied with pain relief from the treatment after $1 \mathrm{~h}$.

Vitamin K3 was used because it is and has been the agent used in the Menstrual Disorder Centre at the Obstetrics and Gynecology Hospital for treatment of primary dysmenorrhoea. Vitamin K3 ampoules were prepared by the Shanghai \#1 Biochemical and Pharmaceutical Company, Ltd. The investigators visited the company in January 2004 to specify a design for blinding (packaging, coding and labelling) of the ampoules. Vitamin K3 and saline ampoules looked identical. The injection site was specified on the ampoules.

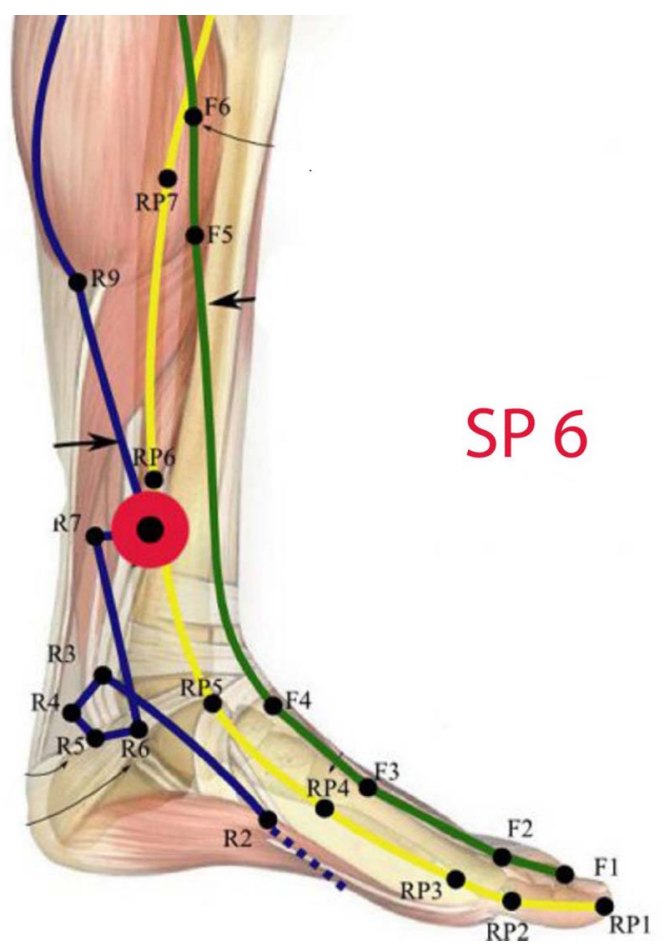

Figure 1 In two of the groups injections were in the Spleen 6 (SP6)/San Yin Jiao (SYJ) acupuncture point near the ankle on both legs. In the third group the injection site was near but not on SP6.

The randomisation list was created by a biostatistician, who went to the certified research pharmacy, which encapsulated the agents to blind the treatment and then sent the ampoules to the research site. Eligible consenting participants were assigned to a group at the treatment visit according to a randomly permuted block design with blocks of size 6 to ensure equal allocation of patients to each treatment group. The randomisation scheme was generated and held by the study biostatisticians.

Physicians were blinded as to saline and vitamin K3, but they knew whether they were injecting into the acupuncture point or near the acupuncture point. Thus, study physicians knew when they were administering to group B, but they could not differentiate the treatments in groups $\mathrm{A}$ and $\mathrm{C}$. Patients presumably did not know the difference between the acupuncture point and near the acupuncture point and were blinded as to the agent in all three groups. Evaluators (study staff collecting pain measures and other study data) did not know the group assignment of participants.

Baseline data were collected following consent and prior to treatment. The primary outcome, change in pain intensity at 0 and $60 \mathrm{~min}$, was recorded on an 11-unit Numeric Rating Scale (NRS) from 0 to 10 with 0 being no pain and 10 being the worst pain the participant could imagine. Pain scores were obtained at baseline and at 2, 5, 30 and $60 \mathrm{~min}$ post-injection. A secondary outcome was the consumption of analgesic 
tablets during the menstrual cycle before and after the injection treatment. Menstrual-related symptoms, such as hours in bed and time lost from school or work, were recorded at baseline and after treatment. Participants' menstrual distress was evaluated after each menstrual cycle on two different scales: Cox Retrospective Symptom Scale (over 6 cycles) and Moos Menstrual Distress Questionnaire (MMDQ) (over 2 cycles)..$^{20} 21$ The Cox scale included 17 symptoms commonly associated with menstrual distress. Each of these was rated by the participant on both intensity and duration with a range from 0 (not perceptible) to 4 (extremely upsetting). The MMDQ assessed six symptoms: muscle stiffness, headache, cramps, backache, fatigue and general aches and pains-each on a range of 0 (not present) to 4 (severe). A questionnaire to assess patients' expectation about group assignment and attitude towards participation in the research study was also administered.

The trial was conducted in two phases. The first phase was conducted as an exploratory pilot with 12 participants randomised to each of the three groups. The results of the pilot were positive, with the effect size for group $\mathrm{C}$ exceeding the hypothesised clinical effect when compared with the other groups. For the second phase, the investigators conducted a power calculation based on the effect sizes of the primary outcome from the pilot phase. Using a Bonferroni correction for multiple testing, we estimated that with 26 patients in each group, we could detect change in mean pain scores of 2 units with SDs of 2.0 between groups at $\alpha=0.05$ and $95 \%$ power. A stratified analysis was planned to accommodate the two phases of the randomised study.

Continuous variables were summarised by descriptive statistics: mean, SD, median and range. Categorical variables were summarised in terms of frequencies and percentages and groups were tested to see if they differed on population characteristics. Initially, results from the two phases of the trial were tested in stratified analyses, however, the results were parallel and homogeneous with no significant difference between the stratified and unstratified results, so the data were then combined in the analysis for reporting. Treatment differences between the three groups were compared both in marginal-effect and mixed-effect models using Stata V.11 (College Station, Texas, USA). A linear mixed-effects model was applied for the primary outcome, where pain scores at time 0 (baseline) and $60 \mathrm{~min}$ were the two response variables (per participant), each of which had its own interceptor representing the average pain level at that time point. ${ }^{22}{ }^{23}$ The observed covariates were treatment modalities and each individual was assigned with an unobserved random effect. The treatment effect is the difference at the $60 \mathrm{~min}$ time point between the two arms under consideration. Significance levels were obtained and adjusted for multiple comparisons using Scheffe and Tukey-Kramer methods. ${ }^{22}$ Analysis of variance (ANOVA) was used to compare mean differences both (1) BETWEEN and (2) WITHIN groups on the secondary outcomes of the Cox and MMDQ scores for cycles 1 and 2 and also for cycles 1 and 7 .

\section{RESULTS}

\section{Baseline data}

The study initiated in October 2004 and ended in June 2010. Phase I was completed in 2 years as it was National Institutes of Health (NIH) funded and physicians had release time to conduct the study: phase II took four additional years to complete. Young Chinese women presenting with severe pelvic pain to an urban hospital clinic who were willing to be randomised to active treatments were screened for eligibility and signed consent forms. The parents of girls under 18 also consented to have their children participate. Of the 124 women screened, 82 enrolled in the study and were randomised, 80 of whom completed all study visits (see figure 2). Two women withdrew after they signed consent forms and were randomised, because they or their parents changed their minds about participation in a research study. The baseline demographic characteristics and menstrual cycle profiles of the women did not differ between the three groups (see table 1).

Reduction of pain: When treated with an acupuncture point injection of vitamin K3 in SP6 (group C), participants had an average 6.6 point decrease in pain on the NRS (from 8.0 to $1.5, \mathrm{p}<0.001$ ) within $60 \mathrm{~min}$ after injection. The average pain decreased by 4.9 points (from 7.9 to $3.0, \mathrm{p}<0.001)$ in the saline acupuncture point injection group (group A) and 4.7 points (from 8.0 to 3.3, $\mathrm{p}<0.001)$ in the vitamin K3 deep muscle injection group (group B) (see figure 3 ).

Difference in group effects: On average, the hypothesised optimal treatment reduced pain by 1.6 and 1.7 units more than in the other groups. Changes in NRS measured pain between group A (active control) and group $\mathrm{B}$ (active control) were less than 1 unit $(-0.71$, CI -1.37 to -0.05$)$ and not significant, but the differences in average scores between group $\mathrm{C}$ (hypothesised to be optimal) and both group A (1.11, CI 0.45 to 1.78$)$ and group B (1.82, CI 1.45 to 2.49) were statistically significant in the unadjusted and adjusted mixed-effects models (see table 2).

Secondary outcomes were the effects on pain intensity and duration of menstrual symptoms for six follow-up cycles: both within-group/between-cycle and mean differences between groups were compared. Menstrual distress was reduced in all post-treatment cycles within all groups. For the two cycles ( 2 and 7 ) we compared with baseline, all groups had significant differences $(\mathrm{p}<0.001)$ between the baseline cycle (cycle 1$)$ and the post-treatment cycle (cycle 2) on the Cox Duration and Cox Intensity scores. All groups also showed a significant difference $(p<0.001)$ between the baseline scores of menstrual distress and cycle 7 scores at the 6-month follow-up report (see table 3). On the long-term measures, significant differences of paired comparisons were 
Figure 2 Flow of participants through the study (SP6, Spleen 6; SYJ, San Yin Jiao).

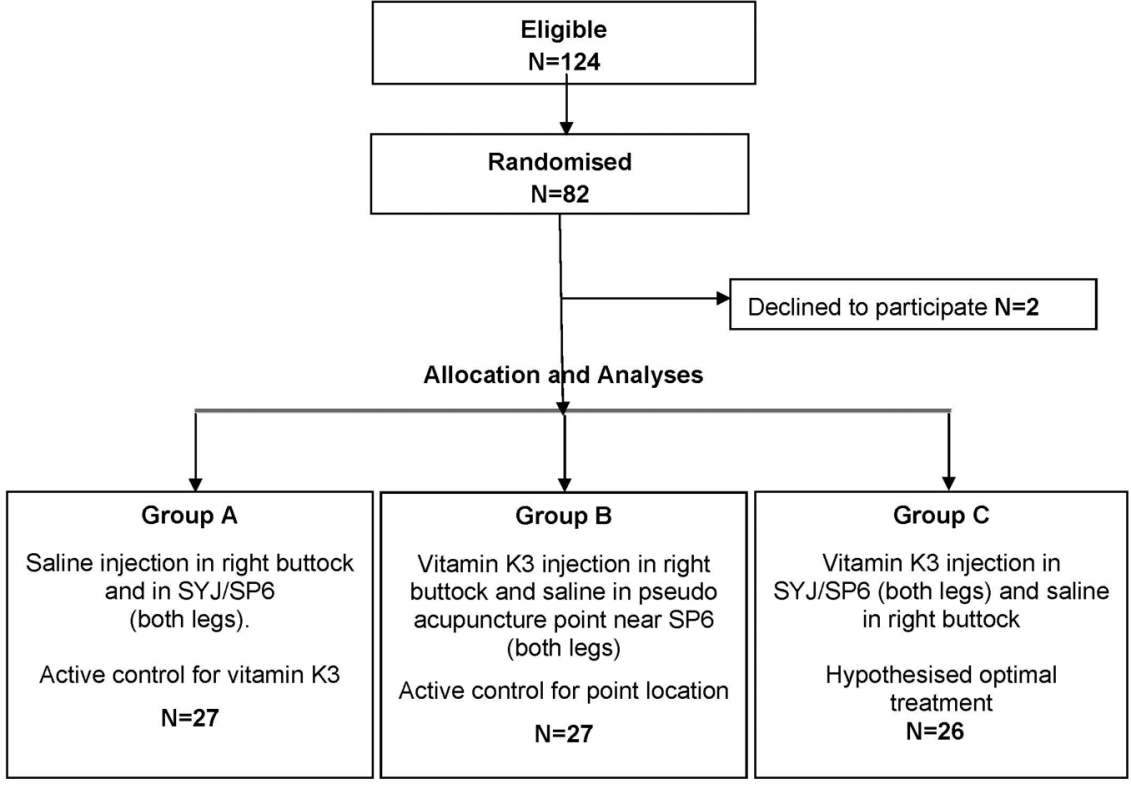

not observed between groups (cycles 1 and 2 and cycles 1 and 7 ) on the Cox Duration and Intensity measures. A significant difference however $(p<0.001)$ was observed between groups on the severity of the six symptoms as measured by the MDDQ cycles 1 and 2 .

Sixty minutes after the treatment and in the follow-up cycles, participants were free to take oral rescue medication (naproxen) if they felt it to be necessary. One tablet of $250 \mathrm{mg}$ of naproxen is a standard treatment for pelvic pain at the Menstrual Disorder Centre. Seven participants in group B, three participants in group A and two participants in group $\mathrm{C}$ asked for and received $250 \mathrm{mg}$ naproxen orally more than $1 \mathrm{~h}$ after the injection treatment and after their pain measurements were recorded. Analgesic tablet consumption during each menstruation period was recorded and is detailed in table 4 . In all follow-up cycles, all three groups consumed fewer oral analgesic tablets compared with what they reported at baseline.

Adverse events reported by four participants (2 in group $\mathrm{A}$, and 1 in groups $\mathrm{B}$ and $\mathrm{C}$ ) included mild discomfort at the injection site that was self-limited and did not require additional medication. Follow-up surveys included questions to assess feasibility of study procedures and expectancy among participants. After participating in the study, the majority of participants $(65.0 \%)$ found the study procedures very or somewhat satisfactory. Thirty-five per cent found them somewhat unsatisfactory but none found them very unsatisfactory, and all participants thought that their participation would help other women with menstrual pain.

Participants were asked whether they thought they had received injection at the true acupuncture point and whether they had received vitamin K3. The majority

\begin{tabular}{|c|c|c|c|c|c|}
\hline & & $\begin{array}{l}\text { Group A } \\
\text { Control for } \\
\text { vitamin K } \\
n=27 \\
\text { Mean } \pm S D\end{array}$ & $\begin{array}{l}\text { Group B } \\
\text { Control for } \\
\text { point location } \\
\mathrm{n}=27 \\
\text { Mean } \pm S D\end{array}$ & $\begin{array}{l}\text { Group C } \\
\text { Standard of care } \\
\text { menstrual } \\
\text { disorder centre } \\
\mathrm{n}=26 \\
\text { Mean } \pm \text { SD }\end{array}$ & $\begin{array}{l}\text { Difference } \\
\text { between } \\
\text { groups } \\
\text { p Value }\end{array}$ \\
\hline Age at start of study & Years & $22.5 \pm 2.2$ & $20.5 \pm 3.2$ & $20.7 \pm 2.9$ & 0.098 \\
\hline Age at menarche & Years & $14.3 \pm 1.1$ & $13.7 \pm 1.0$ & $13.3 \pm 1.2$ & 0.075 \\
\hline Shortest cycle & Days & $26.5 \pm 2.5$ & $26.8 \pm 3.2$ & $26.5 \pm 2.6$ & 0.935 \\
\hline Longest cycle & Days & $33.2 \pm 4.6$ & $32.1 \pm 3.4$ & $31.9 \pm 4.0$ & 0.652 \\
\hline Duration of menstrual flow & Days & $5.7 \pm 0.9$ & $5.9 \pm 1.0$ & $5.7 \pm 1.0$ & 0.763 \\
\hline Missed school or work & Days & $1.8 \pm 0.4$ & $1.8 \pm 0.4$ & $1.9 \pm 0.5$ & 0.781 \\
\hline Extra time spent in bed & Hours & $5.5 \pm 3.1$ & $7.7 \pm 4.9$ & $7.1 \pm 2.0$ & 0.241 \\
\hline Typical worst pain* & NRS† 0-10 & $7.88 \pm 0.62$ & $8.20 \pm 0.41$ & $8.00 \pm 0.65$ & 0.953 \\
\hline
\end{tabular}


Figure 3 Pain intensity at baseline and in the first hour after treatment (SP6, Spleen 6).

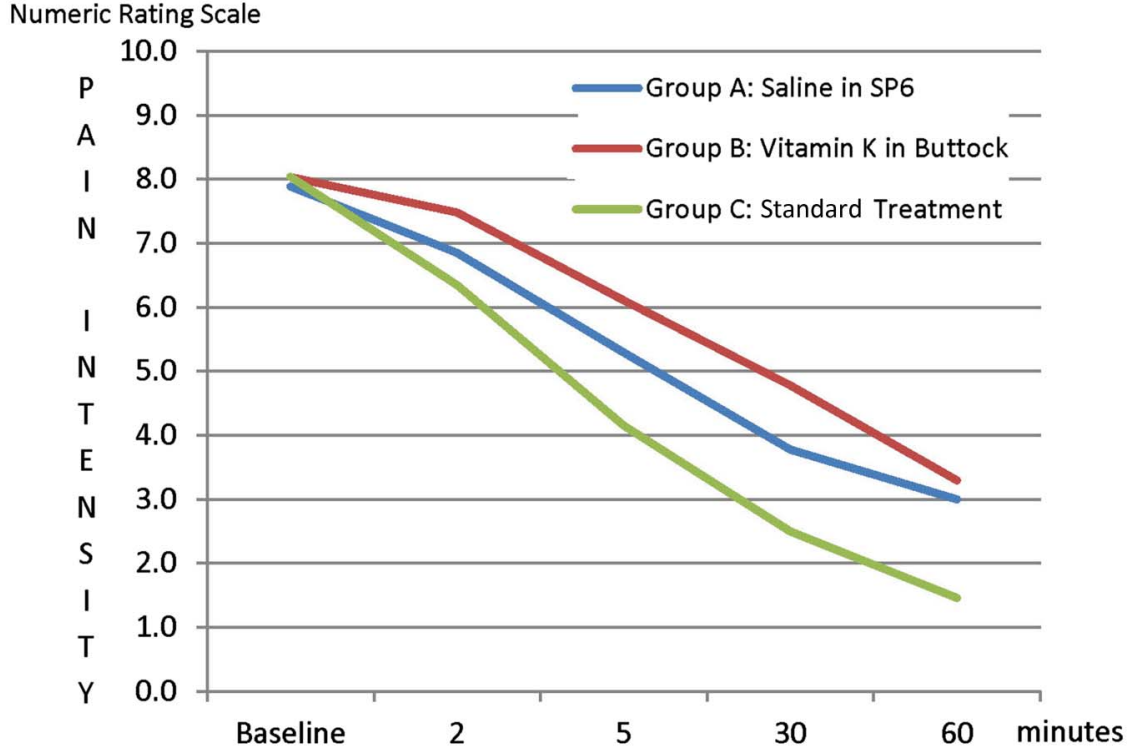

indicated that they did not know which treatment they received $(68.8 \%$ and $57.5 \%)$ and the majority of the remainder thought they had received injection at the true acupuncture point $(30.0 \%)$ as well as vitamin $\mathrm{K} 3$ $(36.3 \%)$. Only a small proportion thought they had received a control treatment: $1.3 \%$ thought they had received an injection near the acupuncture point and $6.3 \%$ thought they had received saline. These results suggest that the blinding procedures were effective.

\section{DISCUSSION}

All three active treatments reduced menstrual cycle pain rapidly (within an hour) with minimal side effects, but acupuncture point injection with vitamin K3 in SP6 was found to be optimal, producing a greater clinical and statistically significant reduction in pain. One to two units on the NRS for pain has been described as clinically significant by studies designed to evaluate unit scores with clinical improvement. ${ }^{24} 25$ Acupuncture point injection with vitamin $\mathrm{K} 3$ provided rapid relief of severe as well as moderate pain due to dysmenorrhoea. Effective treatment can be administered in a highvolume hospital clinic and may be especially useful to women whose pain is unmitigated by other methods. No differences between groups were found on long-term effects, but at the end of the study, participants in all active treatment groups endured significantly less pain over a reduced duration and reported fewer activity restrictions, fewer hours in bed and the consumption of fewer drugs for menstrual pain over the course of six cycles than at baseline, indicating the value of injection treatments for primary dysmenorrhoea. All three treatments reduced pain rapidly during the treatment cycle, yet the treatment in standard use at the clinic, injection of vitamin K3 in SP6, was clearly more effective than the other two treatments on the primary short-term outcome.

The pattern of reduction of menstrual distress over the follow-up cycles is consistent. Women in all of the treatment groups experienced long-term relief. Differences between the three groups on the secondary long-term outcomes of menstrual distress were not significant. Patterns of analgesic use may interact with scores of menstrual distress. In this study, group B, for example, consumed more analgesics than the other two groups. Future research should take this into account when building analytic models. The sensitivity of the Cox and MMDQ scales over the long follow-up period is

Table 2 Pain intensity at baseline and in the first hour after treatment

\section{Overall model F-value $=14.47(p<0.001)$}

Mixed-effect model for different treatment groups

\begin{tabular}{llllllr}
\hline $\begin{array}{l}\text { Treatment } \\
\text { groups }\end{array}$ & $\begin{array}{l}\text { Mean } \\
\text { difference }\end{array}$ & SE & T-value & $\begin{array}{l}\text { Unadjusted } \\
\text { significance }\end{array}$ & $\begin{array}{l}\text { Scheffe } \\
\text { adjusted }\end{array}$ & $\begin{array}{c}\text { Tukey-Kramer } \\
\text { adjusted }\end{array}$ \\
\hline A vs B & -0.708 & 0.338 & -2.095 & 0.037 & 0.113 & 0.093 \\
A vs standard & 1.113 & 0.34 & 3.269 & 0.001 & 0.005 & 0.003 \\
B vs standard & 1.821 & 0.341 & 5.341 & $<0.001$ & $<0.001$ & $<0.001$ \\
\hline
\end{tabular}

Group A: Saline in Spleen 6 (SP6) bilaterally and in the buttock (active control for vitamin K).

Group B: Vitamin K off-point bilaterally and in the buttock (active control for point location).

Group C: Vitamin K in SP6 bilaterally and in the buttock (standard at the Menstrual Disorder Centre). 
Table 3 Menstrual distress before and after injection treatment and during follow-up cycles

\begin{tabular}{|c|c|c|c|c|c|c|}
\hline \multirow[b]{2}{*}{ Study period } & \multirow{2}{*}{$\begin{array}{l}\text { Cox duration* } \\
\text { Mean }\end{array}$} & \multirow{2}{*}{$\begin{array}{l}\text { Cox intensity* } \\
\text { Mean }\end{array}$} & \multirow{2}{*}{$\begin{array}{l}\text { MMDQ* } \\
\text { Mean }\end{array}$} & \multirow{2}{*}{$\begin{array}{l}\text { Daily activity } \\
\text { restriction } \\
\text { Per cent }\end{array}$} & \multicolumn{2}{|c|}{$\begin{array}{l}\text { Increased } \\
\text { hours in bed }\end{array}$} \\
\hline & & & & & Mean & SD \\
\hline \multicolumn{7}{|l|}{ Baseline cycle 1} \\
\hline Group A (control for vitamin K) & 1.42 & 1.19 & 1.20 & 96.30 & 6.52 & 3.95 \\
\hline Group B (control for point location) & 1.40 & 1.18 & 1.38 & 100.00 & 6.67 & 4.26 \\
\hline Group C (standard†) & 1.29 & 1.18 & 1.34 & 100.00 & 7.58 & 5.38 \\
\hline \multicolumn{7}{|l|}{ Treatment cycle 2} \\
\hline Group A (control for vitamin K) & $0.84 \ddagger$ & $0.68 \ddagger$ & 0.69 & 59.26 & 3.60 & 1.35 \\
\hline Group B (control for point location) & $0.93 \ddagger$ & $0.68 \ddagger$ & 0.82 & 74.07 & 5.40 & 2.80 \\
\hline Group C (standard†) & $0.83 \ddagger$ & $0.69 \ddagger$ & $0.69 \S$ & 61.54 & 4.57 & 1.81 \\
\hline \multicolumn{7}{|l|}{ Cycle 3} \\
\hline Group A (control for vitamin K) & 0.85 & 0.66 & $\mathrm{n} / \mathrm{a}$ & 65.38 & 4.33 & 1.30 \\
\hline Group B (control for point location) & 0.93 & 0.76 & $\mathrm{n} / \mathrm{a}$ & 62.96 & 5.00 & 1.85 \\
\hline Group C (standard $\dagger)$ & 0.73 & 0.57 & $\mathrm{n} / \mathrm{a}$ & 50.00 & 5.13 & 2.47 \\
\hline \multicolumn{7}{|l|}{ Cycle 4} \\
\hline Group A (control for vitamin K) & 0.90 & 0.65 & $\mathrm{n} / \mathrm{a}$ & 42.31 & 7.50 & 5.73 \\
\hline Group B (control for point location) & 0.82 & 0.67 & $\mathrm{n} / \mathrm{a}$ & 55.56 & 5.00 & 1.61 \\
\hline Group C (standard †) & 0.76 & 0.64 & $\mathrm{n} / \mathrm{a}$ & 46.15 & 5.11 & 1.76 \\
\hline \multicolumn{7}{|l|}{ Cycle 5} \\
\hline Group A (control for vitamin K) & 0.77 & 0.52 & $\mathrm{n} / \mathrm{a}$ & 42.31 & 7.67 & 5.99 \\
\hline Group B (control for point location) & 0.78 & 0.66 & $\mathrm{n} / \mathrm{a}$ & 55.56 & 5.11 & 1.76 \\
\hline Group C (standard†) & 0.76 & 0.57 & $\mathrm{n} / \mathrm{a}$ & 57.69 & 3.60 & 0.52 \\
\hline \multicolumn{7}{|l|}{ Cycle 6} \\
\hline Group A (control for vitamin K) & 0.84 & 0.66 & $\mathrm{n} / \mathrm{a}$ & 61.54 & 8.14 & 3.18 \\
\hline Group B (control for point location) & 0.93 & 0.77 & $\mathrm{n} / \mathrm{a}$ & 66.67 & 5.45 & 1.81 \\
\hline Group C (standard†) & 0.87 & 0.63 & $\mathrm{n} / \mathrm{a}$ & 57.69 & 4.91 & 1.38 \\
\hline \multicolumn{7}{|l|}{ Cycle 7} \\
\hline Group A (control for vitamin K) & $0.79 \rrbracket$ & $0.60 \uparrow$ & $\mathrm{n} / \mathrm{a}$ & 57.69 & 4.89 & 2.03 \\
\hline Group B (control for point location) & $0.98 \emptyset$ & $0.78 \rrbracket$ & $\mathrm{n} / \mathrm{a}$ & 59.26 & 5.25 & 1.82 \\
\hline Group C (standard $\dagger)$ & 0.779 & $0.61 \rrbracket$ & $\mathrm{n} / \mathrm{a}$ & 50.00 & 5.50 & 2.39 \\
\hline
\end{tabular}

*Although all three groups experienced reduction in menstrual distress on the Cox and MMDQ scales between baseline and cycle 2 and between baseline and cycle 7, the only significant difference in group means was on the MMDQ scale between baseline and cycle 2 . †Standard treatment at the Menstrual Disorders Centre, Obstetrics and Gynecology Hospital, Shanghai. \#Significant difference $(p<0.001)$ between baseline cycle and treatment cycle within group paired comparisons. $\S$ Significant difference $(p<0.001)$ between groups $A$ and $C$ and between groups $B$ and $C$ on menstrual distress. ๆSignificant difference $(p<0.001)$ between baseline cycle and last follow-up cycle within group paired comparisons. MMDQ, Moos Menstrual Distress Questionnaire; n/a, not applicable.

unknown. Both instruments were administered with the idea of validating results from one to the other and determining the best instrument for future research. The MMDQ scale may be more sensitive than the Cox scale for long-term follow-up assessment.

The overall reduction of menstrual distress and the decrease in the need for analgesics across groups during the follow-up period is notable. Whether these effects are due to the treatment or to the natural course of dysmenorrhoea over 6 months is unknown, but merits further study. Differences between the effects of the three treatments on menstrual distress in subsequent cycles were more variable than the short-term pain outcome and may be obscured by the use of pain medications and other home remedies which participants were free to employ during the long follow-up period.

The three-arm study was designed to control for multiple effect pathways, and therefore the results support the injection site (an acupuncture point) as a crucial aspect of optimal pain relief. Little information exists about any specific mechanism of acupuncture's effect on menstrual pain, and while acupuncture's interruption of pain pathways has been investigated since $1979,{ }^{26}$ much remains unknown about the cause and magnitude of the effects of acupuncture, as placebo-controlled trials of manual treatments are difficult to evaluate. Many studies of acupuncture do not find significant difference of effects for off-point and on-point stimulation with acupuncture needles. ${ }^{27}{ }^{28}$ Point injection may have different effects than classical acupuncture, and measurement of outcomes may not have been sensitive enough in some previous studies to determine optimal effects. ${ }^{29}$ Benefits of acupuncture point injection may include rapid and substantial pain relief and prolonged effects across a variety of pain conditions. ${ }^{30-32}$ The three injection treatments administered in this study worked rapidly, perhaps quicker than NSAIDs, which optimises at $1-2 \mathrm{~h},{ }^{33} 34$ and our study indicates that the treatment may have long-term 
Table 4 Analgesic tablet consumption at baseline and six follow-up cycles

\begin{tabular}{|c|c|c|c|c|c|c|}
\hline & \multicolumn{2}{|c|}{$\begin{array}{l}\text { Group A } \\
\text { (Control for } \\
\text { vitamin K) }\end{array}$} & \multicolumn{2}{|c|}{$\begin{array}{l}\text { Group B } \\
\text { (Control for } \\
\text { point } \\
\text { location) }\end{array}$} & \multicolumn{2}{|c|}{$\begin{array}{l}\text { Group C } \\
\text { (Standard) }\end{array}$} \\
\hline & Mean & (SD) & Mean & (SD) & Mean & (SD) \\
\hline Baseline & 0.93 & 0.82 & 0.82 & 0.56 & 0.89 & 0.77 \\
\hline Cycle 2 & 0.22 & 0.42 & 0.41 & 0.57 & 0.27 & 0.45 \\
\hline Cycle 3 & 0.11 & 0.32 & 0.33 & 0.48 & 0.35 & 0.49 \\
\hline Cycle 4 & 0.22 & 0.42 & 0.41 & 0.5 & 0.27 & 0.45 \\
\hline Cycle 5 & 0.22 & 0.42 & 0.48 & 0.5 & 0.35 & 0.49 \\
\hline Cycle 6 & 0.33 & 0.48 & 0.48 & 0.58 & 0.23 & 0.43 \\
\hline Cycle 7 & 0.22 & 0.42 & 0.33 & 0.48 & 0.23 & 0.43 \\
\hline
\end{tabular}

effects on menstrual distress of up to 6 months. An effect of up to 3 months has been observed in at least one previous study. ${ }^{14}$ Although this treatment cannot be selfadministered, it does not require dosing prior to the onset of worst symptoms, as NSAIDs do, and nor does it require daily dosing as OCs do.

Our results also indicate that vitamin K3 may have some role in amelioration of menstrual pain, although the mechanism is as yet unclear, as none of the forms of vitamin $\mathrm{K}$ are known to have analgesic effects. At least one form of vitamin $\mathrm{K}$ is absorbed through this treatment: increased concentrations of vitamin K1 (phylloquinone) following injection at SP6 were observed in a study of four participants with primary dysmenorrhoea. ${ }^{18}$ Analysis of plasma phylloquinone indicated non-detectable concentrations before acupuncture point injection with vitamin $\mathrm{K} 1$, which increased up to $425 \mathrm{nmol} / \mathrm{L}, 20-48 \mathrm{~h}$ following injection. The vitamin $\mathrm{K}$ profile of women with menstrual cycle disorders may be worth exploring in a nutritional approach to dysmenorrhoea prevention.

The use of vitamin K3 (menadione) for this treatment is routine at the Menstrual Disorder Centre at the Obstetrics and Gynecology Hospital where this study was conducted. ${ }^{14}{ }^{16}$ Studies in the USA and Europe have tested alternate forms of vitamin K. Vitamin K1 (phylloquinone) and K4 (menaquinone-4) were chosen over vitamin K3 because of availability in the clinical setting and because vitamin $\mathrm{K} 1$ has a better safety profile than vitamin K3. ${ }^{35}$ These studies also found that menstrual pain and distress were relieved. ${ }^{14}{ }^{15}{ }^{17}$ Little is known about vitamin K's role in ameliorating symptoms of dysmenorrhoea or difference in effect in the administration of the various forms of vitamin K. NSAIDs alleviate the pain of menstrual cramps through their effect on prostaglandin levels. One study that measured prostaglandin levels after acupuncture using the point SP6 did not find that the treatment reduced them. ${ }^{6}$ To the best of our knowledge, studies of vitamin $\mathrm{K}$ and prostaglandin levels have not been reported. However, vitamin $\mathrm{K}$ is being explored for its potential role in reducing proinflammatory cytokines. ${ }^{36}$ Vitamin $\mathrm{K}$ is typically used clinically to abet blood clotting and, recently, has been studied in relation to bone health. Animal studies have shown that ovary concentrations of vitamin $\mathrm{K}$ are higher than in other organs, ${ }^{37}$ and researchers have suggested that vitamin $\mathrm{K}$ may play a role in reproductive endocrinology. ${ }^{38}$

Our overarching goal was to create a research agenda leading to a larger comparative effectiveness study conducted by international collaborators in three countries, and the study reported here was conceived as necessary preliminary research to an international trial of acupuncture point injection of vitamin K in SP6 vs NSAIDs, which would be executed in the three countries where pilot research had been successfully conducted. Studies in Europe, North America and Asia have indicated that this treatment reduces menstrual pain in young women with primary dysmenorrhoea, in a variety of outpatient settings in diverse cultures. The results of these studies as a group indicate that various forms of vitamin $\mathrm{K}(\mathrm{K} 1, \mathrm{~K} 4$ and K3) can produce pain relief when utilised with acupuncture point injection and have also indicated that the treatment is adaptable to various clinical settings (public health clinics, private practice, hospital outpatient facilities) and acceptable to young women in North America and Europe, as well as in China. ${ }^{14-17}$ The study reported here is one of two known randomised studies of the treatment ${ }^{17}$ and has the largest sample size and most rigorous design of all known studies on the treatment. Results reported here also suggest that both the agent and site of injection influence the effect size of the short-term pain reduction measures.

\section{Study limitations}

The study setting may limit generalising the results. Expectation by Chinese women that acupuncture will provide pain relief may have influenced reports of pain reduction. However, this limitation is mitigated by the research efforts of international collaborators who have implemented the clinical protocol in a variety of countries and settings. The hypothesised optimal treatment in this study has been tested on Chinese, American and Italian women with primary dysmenorrhoea and has reduced menstrual pain in young women in public health clinics, private practice and hospitals. ${ }^{14} 1517$ The study was implemented in two phases under two different randomisation schemes and data from the first phase were analysed prior to the implementation of the second phase of the study. Initially, the study team completed a pilot study of 36 patients and analysed the data. Since the hypothesised results were positive and the effect size was robust, the study was powered and data were collected for another 44 patients for more stable results. We performed analysis stratified by the two phases and found no significant differences in results by phase, which supports our methodological approach of analysing and reporting the combined results. Menstrual distress scores on two different scales were compared 
with Student $\mathrm{t}$ tests and not in an adjusted model for multiple comparisons.

The study took over 6 years to complete in a very busy outpatient clinic. Recruitment during the second phase of the study became ever more challenging. Whereas the first phase received external funding, in the second phase the funding was greatly reduced, limiting the release time of the study physicians and making the 6 months of follow-up data collection difficult. Scheduling difficulties also affected recruitment, as it is important for participants to be treated within 2 days of onset of menses and at the height of their pain. However, this was not always possible, and this limited participation in the study.

\section{CONCLUSION}

Our study reports the effect of acupuncture point injection of vitamin K3 on the pain of dysmenorrhoea in young Chinese women. It extends the work of previous single-arm and cross-over studies through the implementation of a randomised parallel group design, and provides substantial evidence that the treatment provides pain relief for women with painful periods who have not been able to obtain satisfactory relief by any other method. Study results also indicate that the site of injection and the specific agent of vitamin $\mathrm{K}$, specifically vitamin $\mathrm{K} 3$, are crucial to the optimal treatment of pain due to primary dysmenorrhoea.

\section{Author affiliations}

${ }^{1}$ Richard \& Hinda Rosenthal Center for Complementary and Alternative Medicine Research, College of Physicians and Surgeons, Columbia University, New York, New York, USA

${ }^{2}$ Institute of East West Medicine, New York, New York, USA

${ }^{3}$ Obstetrics and Gynecology Hospital, Fudan University, Shanghai, Shanghai, China

${ }^{4}$ Agenzia Sanitaria e Sociale Regionale dell'Emilia Romagna, (Healthcare and Social Agency of Emilia Romagna Region), Viale Aldo Moro, 21, 40127 Bologna, Italy

${ }^{5}$ Department of Anesthesiology, Pain and Perioperative Medicine, Stanford University School of Medicine, Palo Alto, California, USA

${ }^{6}$ Department of Statistics, Columbia University, New York, New York, USA

${ }^{7}$ Department of Statistics, School of Public Health, Fudan University,

Shanghai, Shanghai, China

${ }^{8}$ Osher Center for Integrative Medicine, University of California at

San Francisco, San Francisco, California, USA

Contributors CW contributed to the study design, project management, data analysis and interpretation, and wrote the first draft of the manuscript. LW, WJZ and SQG contributed to the study design and procedures, data acquisition and quality, study administration and interpretation of results. FC contributed to the study design and interpretation of results. FK obtained the study funding and participated in the administration and data acquisition. ZY was responsible for the power calculation, statistical analysis and interpretation and data monitoring. NQZ was responsible for the randomisation, blinding, statistical analysis and interpretation and data monitoring. MTC performed the statistical analysis and interpretation. JY developed the treatment protocol and provided the pilot data.

Funding Funding was provided by an NIH/NCCAM Planning Grant for International Research on Chinese Medicine and Women's Health (R21-AT01957), the Obstetrics and Gynecology Hospital, Shanghai, China and the Institute of East/West Medicine, New York, New York, USA. Additional funding was received through NIH/NCCAM (K01AT006545).
Disclaimer The contents of this paper are solely the responsibility of the authors and do not necessarily represent the official views of the NIH or NCCAM or other funders.

Competing interests None declared.

Ethics approval Columbia University Medical Center IRB, New York, USA: Obstetrics and Gynecology Hospital Medical Ethics Board, Shanghai, China.

Provenance and peer review Not commissioned; externally peer reviewed.

Data sharing statement No additional data are available.

Open Access This is an Open Access article distributed in accordance with the Creative Commons Attribution Non Commercial (CC BY-NC 4.0) license, which permits others to distribute, remix, adapt, build upon this work noncommercially, and license their derivative works on different terms, provided the original work is properly cited and the use is non-commercial. See: http:// creativecommons.org/licenses/by-nc/4.0/

\section{REFERENCES}

1. Harlow SD, Park M. A longitudinal study of risk factors for the occurrence, duration and severity of menstrual cramps in a cohort of college women. Br J Obstet Gynaecol 1996;103:1134-42.

2. Klein JR, Litt IF. Epidemiology of adolescent dysmenorrhea. Pediatrics 1981;68:661-4.

3. Dawood MY. Dysmenorrhea. Clin Obstet Gynecol 1990;33:168-78.

4. Latthe PM, Champaneria R, Khan KS. Dysmenorrhoea. BMJ Clin Evid 2011;2011:pii: 0813.

5. Marjoribanks J, Proctor ML, Farquhar C. Nonsteroidal anti-inflammatory drugs for primary dysmenorrhoea. Cochrane Database Syst Rev 2003; (4):CD001751.

6. Shi GX, Liu CZ, Zhu J, et al. Effects of acupuncture at Sanyinjiao (SP6) on prostaglandin levels in primary dysmenorrhea patients. Clin $J$ Pain 2011;27:258-61.

7. Davis AR, Westhoff CL. Primary dysmenorrhea in adolescent girls and treatment with oral contraceptives. J Pediatr Adolesc Gynecol 2001;14:3-8.

8. Dawood MY. Ibuprofen and dysmenorrhea. Am J Med 1984;77:87-94.

9. Liu CZ, Xie JP, Wang LP, et al. Immediate analgesia effect of single point acupuncture in primary dysmenorrhea: a randomized controlled trial. Pain Med 2011;12:300-7.

10. Chen $\mathrm{CH}$. Acupuncture: a comprehensive text, Shanghai college of traditional medicine. Shanghai: Eastland Press, 1981.

11. Strudwick MW, Hinks RC, Choy STB. Point injection as an alternative acupuncture technique-an exploratory study of responses in healthy subjects. Acupunct Med 2007;25:166-74.

12. Yu J. Clinical and experimental study of dysfunctional dysmenorrhea treated by Vit K. New Drugs Clin Rem 1985;5:14-6.

13. Zhao WJ, Weng JE, Yu J. Clinical study of vitamin K3 acupoint injection in treating pelvic pain. Chinese acupuncture \& moxibustion 2000;20:393-4.

14. Wang L, Cardini F, Zhao W, et al. Vitamin K acupuncture point injection for severe primary dysmenorrhea: an international pilot study. Med Gen Med 2004;6:45.

15. Lesi G, Gramenzi A, Gammi L, et al. Vitamin K injection in San Yin Jiao acupoint (SP6) to treat severe primary dysmenorrhoea: results of a pilot study. Eur J Integr Med 2012;4:S51.

16. Zhao WJ, Wang L, Weng JE, et al. Clinical study of vitamin K3 acupoint injection in treating pelvic pain. Chin $J$ Integr Med 2003;9:136-8.

17. Chao $\mathrm{M}$, Wade $\mathrm{C}$, Abercombie $\mathrm{P}$, et al. An innovative acupuncture treatment for primary dysmenorrhea: a randomized, crossover pilot study. Altern Ther 2014;20:45-52.

18. Chao M, Wade C, Booth SL. Plasma phylloquinone concentrations increase following acupoint injection for primary dysmenorrhea. $J$ Acupunct Meridian Stud 2014;7:151-4.

19. Robinson JC, Plichta S, Weisman CS, et al. Dysmenorrhea and use of oral contraceptives in adolescent women attending a family planning clinic. Am J Obstet Gynecol 1992;166:578-83.

20. Cox DJ. Menstrual symptom questionnaire: further psychometric evaluation. Behav Res Ther 1977;15:506-8.

21. Moos $\mathrm{RH}$. The development of a menstrual distress questionnaire. Psychosom Med 1968;30:853-67.

22. Scheffe H. The analysis of variance. New York: Wiley, 1959.

23. Fitzmaurice GM, Laird NM, Ware JH. Applied longitudinal analysis. New York: Wiley, 2004.

24. Todd $\mathrm{KH}$. Clinical versus statistical significance in the assessment of pain relief. Ann Emerg Med 1996;27:439-41. 
25. Farrar JT, Young JP Jr, LaMoreaux L, et al. Clinical importance of changes in chronic pain intensity measured on an 11-point numerical pain rating scale. Pain 2001;94:149-58.

26. Pomeranz B, Cheng R. Suppression of noxious responses in single neurons of cat spinal cord by electroacupuncture and its reversal by the opiate antagonist naloxone. Exp Neurol 1979:64:327-41.

27. McManus CA, Schnyer RN, Kong J, et al. Sham acupuncture devices-practical advice for researchers. Acupunct Med 2007;25:36-40.

28. Park J, Linde K, Manheimer E, et al. The status and future of acupuncture clinical research. J Altern Complement Med 2008:14:871-81.

29. Vickers AJ, Cronin AM, Maschino AC, et al. Acupuncture for chronic pain: individual patient data meta-analysis. Arch Intern Med 2012;172:1444-53.

30. Kwon YB, Kang MS, Kim HW, et al. Antinociceptive effects of bee venom acupuncture (apipuncture) in rodent animal models: a comparative study of acupoint versus non-acupoint stimulation. Acupunct Electrother Res 2001:26:59-68.

31. Lee MS, Pittler MH, Shin BC, et al. Bee venom acupuncture for musculoskeletal pain: a review. J Pain 2008;9:289-97.
32. Park JM, Park SU, Jung WS, et al. Carthami-Semen acupuncture point injection for chronic daily headache: a pilot, randomised, double-blind, controlled trial. Complement Ther Med 2011;19(Suppl 1):S19-25.

33. Marjoribanks J, Proctor M, Farquhar C, et al. Nonsteroidal anti-inflammatory drugs for dysmenorrhoea. Cochrane Database Syst Rev 2010;(1):CD001751.

34. Milsom I, Minic M, Dawood MY, et al. Comparison of the efficacy and safety of nonprescription doses of naproxen and naproxen sodium with ibuprofen, acetaminophen, and placebo in the treatment of primary dysmenorrhea: a pooled analysis of five studies. Clin Ther 2002;24:1384-400.

35. Bennett MI, Smith BH, Torrance N, et al. The S-LANSS score for identifying pain of predominantly neuropathic origin: validation for use in clinical and postal research. J Pain 2005;6:149-58.

36. Booth SL. Roles for vitamin K beyond coagulation. Annu Rev Nutr 2009;29:89-110.

37. Huber AM, Davidson K, O'Brien-Morse ME, et al. Tissue phylloquinone and menaquinones in rats are affected by age and gender. J Nutr 1999;129:1039-44.

38. Truong JT, Booth SL. Emerging issues in vitamin $\mathrm{K}$ research. J Evid Based Complement Altern Med 2011;16:73-9. 